\title{
Radial Line Slot Array Antenna Feeder Design Using Optimization
} Algorithm

\author{
Iliya S. Zakwoi ${ }^{1}$, Soyinka O. Kolawole ${ }^{2}$, Ogbeide O. $\mathrm{K}^{3} \&$ Omeiza I. O. $\mathrm{A}^{4}$ \\ ${ }^{1}$ Department of Electrical and Information Engineering, Landmark University, Nigeria \\ ${ }^{2}$ Department of Mechanical Engineering, Landmark University, Nigeria \\ ${ }^{3}$ University of Benin, Benin City, Edo State, Nigeria \\ ${ }^{4}$ University of Ilorin, Kwara State, Nigeria \\ Correspondence: Iliya Zakwoi, Department of Electrical and Information Engineering, Landmark University, \\ Nigeria. Tel: 234-8161-856-357. E-mail: iliya.solomon@lmu.edu.ng
}

Received: Oct. 24, 2018

doi:10.5539/mas.v13n1p214
Accepted: Nov. 5, 2018

Online Published: December 31, 2018

URL: https://doi.org/10.5539/mas.v13n1p214

\begin{abstract}
High Bandwidth is required today to meet the demands for high data rates needed for future Direct-to Home (DTH) services in Ka-Band. In this work, the Brainstorm Optimization (BSO) method for the feeder architecture design is utilized to meet this need at $28 \mathrm{GHz}$. The BSO is used as tool for the optimization of the beam squinted antenna feeder parameters in terms of air gaps, radial cavity dimensions and dielectric thickness needed for the optimal customization of the conventional Sub-Miniature A (SMA) connector for enhanced bandwidth realization in Ka Band at $28 \mathrm{GHz}$ required for Direct to Home Services (DTH). Solutions values realized from the BSO algorithm using MATLAB computations were included in a visual BASIC program in the macros of CST 2017 microwave suites for pattern generation and antenna radiation characteristics computations A radiation efficiency value of 95.97\% was achieved, an Impedance Bandwidth of $99.61 \%$ and Gain of $23.09 \mathrm{dBi}$ were achieved. Results were compared with earlier studies on $\mathrm{Ka}$ Band at $28 \mathrm{GHz}$ and the results shows an improvement in the bandwidth and directivity values.
\end{abstract}

Keywords: beam squint, bso, bandwidth, efficiency, rlsa-antenna

\section{Introduction}

\subsection{Antenna Feed Parameter Optimization}

Various studies adopted several architectures for the optimization of the RLSA feeder, this was aimed at improving bandwidth, directivity, gain and return loss, notable amongst this studies, is the works of, I. Maina, T. A. Rahman, M. Khalily, and S. I. Zakwoi. (2016), which adopted the use of a syntactic foam and an RT/duroid 5880 dielectric materials for a multilayer feeder design at 28GHz. Good results were obtained. The Radial Line Slot Array (RLSA) Antenna consists of concentric annular rings of slots positioned at an angle adjacent to each other in an array manner and the contributions resulting from the array geometry results in high directivity. It is easy to fabricate, install and also have low cost of production.

Recently the Linearly Polarized Radial Line Slots Array (LP-RLSA) Antenna has demonstrated remarkable potentials capable of replacing the traditional parabolic dish reflector antennas because of its high directivity values recorded by different authors, M. Ando, K. Sakurai, and N, Goto. (1986), P. Davis (2000), P. W. Davis, M. E. Bialkowski, and S. Lucia. (2000). Advancement in the study of this type of antenna has shown different Feeder architecture design aimed at improving desired parameters associated with the RLSA antenna feeding network, notably are the works of, P. W. Davis. (1997), I. Maina, T. A. Rahman, M. Khalily, and S. I. Zakwoi. (2016), P. W. Davis and M. E. B. I. (1999), which suggest some improvements in terms of bandwidth, return loss and directivity values. Satellites launched into space carrying Ka Band Transponders are referred to as high Throughput Satellites (HTS) which requires large bandwidth to meet the demands for high data rates needed for the future Ka band Spectrum for DTH applications as recommended by ITU-R.

The introduction of the BSO algorithm however, brought about the eradication of design complexities and time wastage involved in the selection of optimal feeding parameters for best simulation radiation results. To obtain 
optimal design parameters for the antenna, the basic Brainstorm Optimization algorithm (BSO) developed by, Y. H. Shi. (2011), and its various modifications by scholars, as found in, Z. Zhan, J. Z hang, Y. H. Shi \& H. Liu. (2012), C.H Sun \& H.B Duan (2013), and O.K. Soyinka, H. Duan. (2016), is used in this paper. The BSO process is divided into population initialization, clustering, individual generation, disruption and selection. A description of the pseudo code algorithm is given as follows:

\subsection{Pseudo-Code for the Basic BSO Algorithm}

\section{Step .1 Start.}

Step .2 Population initialization.

1. Counter $=1$.

2. Generate $n$ random potential solutions $x_{i} ; i=1,2,3 \ldots ., n$.

3. Evaluate the fitness of the $n$ individuals.

DO while iter $<$ maximum iteration.

Step .3 Clustering

1. Cluster $n$ individuals into $m$ clusters using clustering algorithm.

2. Rank individuals in each cluster $j$ and select the best individual as cluster center in each cluster (denoted $\left.x_{c}^{j}\right)$. Where $j=1,2,3, \ldots, \mathrm{m}$.

Step .4 Disrupt the cluster center.

1. Randomly generate a value $P_{1}[0,1]$,

2. If $P_{1}<P_{5 a} \quad\left(P_{5 a}\right.$ is a pre-determined probability of 0.2$)$,

3. Randomly select a cluster center $\left(x_{c}\right)$.

4. Randomly generate an individual to replace the selected cluster center.

5. Else, retain the selected cluster center.

Step .5 Updating individuals.

For $x_{i} ; i=1,2,3 \ldots, n$

1. Randomly generate a value $P_{2}[0,1]$,

If $P_{2}<P_{6 b}$ ( $P_{6 b}$ is a pre-determined probability of 0.8 ),

2. Randomly select a cluster with a probability $P_{6 b}$.

3. Randomly generate a value $P_{3}[0,1]$,

If $P_{3}<P_{6 b i i i}$ ( $P_{6 b i i i}$ is a pre-determined probability of 0.4 ),

4. Select the cluster centre and add random noises to generate a new individual.

5. Else, randomly select an individual from this cluster, and add random noises to the individual to generate a new individual.

6. If $P_{2}>P_{6 b}$ randomly select two clusters to generate new individual,

7. Generate a random value $P_{4}[0,1]$.

If $P_{4}<P_{6 c}\left(P_{6 c}\right.$ is a pre-determined probability of 0.5$)$,

Combine the two cluster centres

8. Combine the two cluster centers, and add random values to generate a new individual.

9. Else, randomly select two individuals from each selected cluster, combine them, and add random values to generate a new individual.

Step .6 Compare the newly generated individual with the existing individual having the same individual index, select the better as the new individual.

10. and add random values to generate a new individual.

11. Else, randomly select two individuals from each selected cluster, combine them, and add random values to generate a new individual.

Step .7 Compare the newly generated individual with the existing individual having the same individual index, 
select the better as the new individual.

End for loop.

Counter $=$ counter +1 .

End while loop.

\section{Problem Formulation}

The work seek to obtain optimal angles for $\left(\theta_{T}, \phi_{T}\right)$ and $h$, representing, squint angle in the azimuth direction, the current flow line and the dielectric material thickness respectively. Since the fitness value cannot be negative, the optimization problem is written as:

$$
\min \left(\Im_{\max }=\frac{2 h}{1-\xi \sin \theta_{T} \cos \left(\phi-\theta_{T}\right)}\right)
$$

Subject to:

i. $\quad 0.01 \leq \theta_{T} \leq \pi$

ii. $0.01 \leq \phi_{T} \leq \pi$

iii. $1 \leq h \leq 5$

To solve for the optimal values in the objective function in eq. (1), the parameters in Table 1 are assumed for the BSO algorithm.

Table 1. BSO parameters

\begin{tabular}{lllllllll}
\hline$D$ & $K$ & $P_{5 a}$ & $P_{6 b}$ & $P_{6 i i i}$ & $P_{6 c}$ & Maximum Iteration & $\boldsymbol{\mu}$ & $\sigma$ \\
\hline 2 & 5 & 0.2 & 0.8 & 0.4 & 0.5 & 2000 & 0 & 1 \\
\hline
\end{tabular}

$D$ in table 1 represents the dimension of the search space, $K$ is the cluster size, $P_{5 a}, P_{6 b}, P_{6 i i i}, P_{6 c}$ are predefined probabilities according to the BSO algorithm steps. $\mu$ and $\sigma$ are the mean and standard deviation respectively

\subsection{BSO Optimization Results}

The result of the optimization is shown in Figure 1 and the numerical values of the optimal parameters and fitness is given in table 2 .

Table 2. Optimal design parameters

\begin{tabular}{|c|c|c|c|c|}
\hline Fitness Value & $\theta_{T}(\mathrm{rad})$ & $\phi_{T}(\mathrm{rad})$ & $h(\mathrm{~mm})$ & $\xi_{r}$ \\
\hline 1.3381 & 2.6340 & & 1.00025 & 1.0000 \\
\hline
\end{tabular}

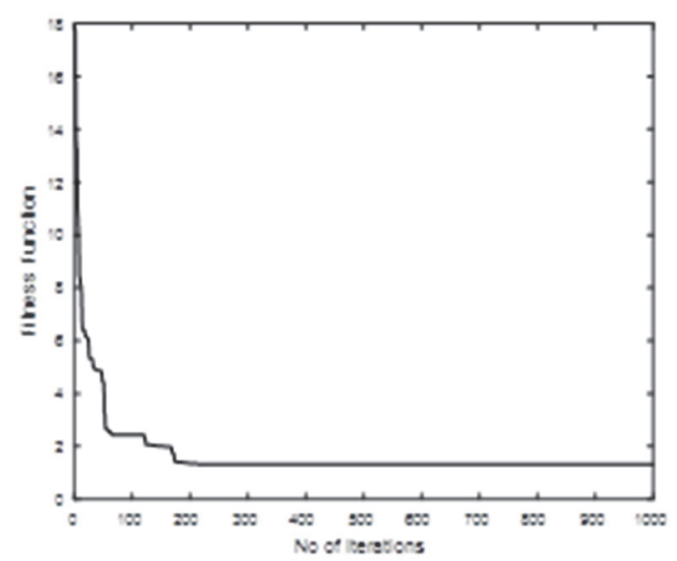

Figure 1. Evolution of fitness curve 


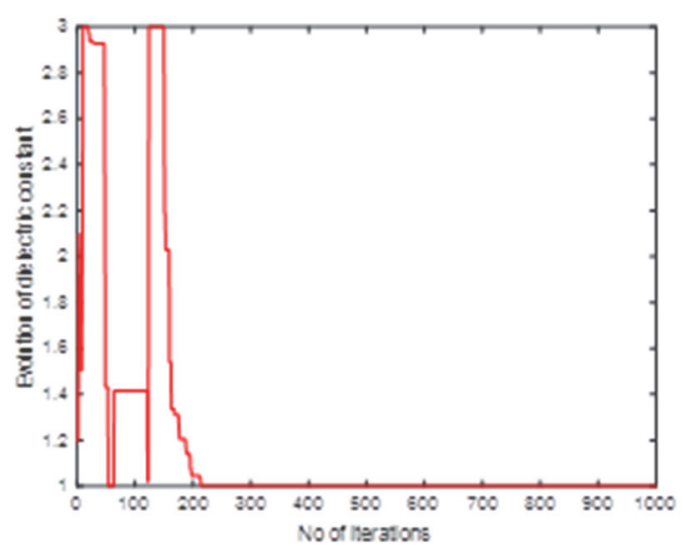

Figure 2. Evolution of dielectric constant

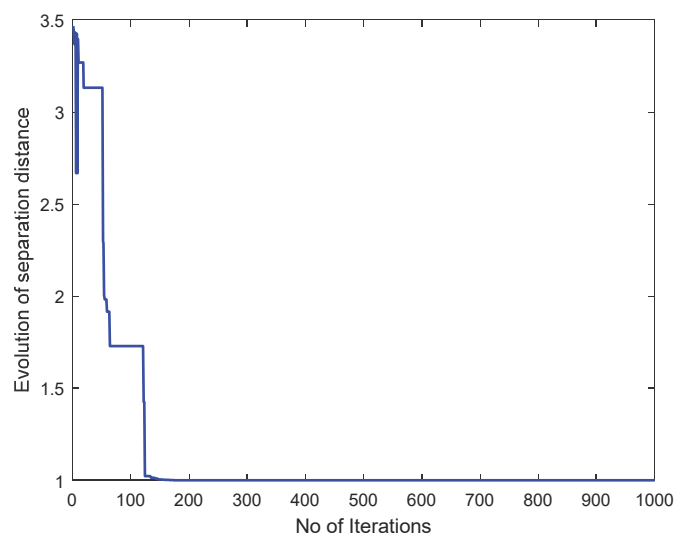

Figure 3. Evolution of separation constant

Figure 1 shows the evolution of the optimization function in eq. (1). The function converges to a minimum value of 1.3381. The corresponding values for the dielectric constant and the separation distance over the number of iterations is shown in figures 2 and 3. The optimal values within the search space begins to converge for the dielectric constant after the $150^{\text {th }}$ iteration; it occurs earlier for the separation distance at the $20^{\text {th }}$ iteration. The plots converge to numerical values of 1.0000 and $1.00025 \mathrm{~mm}$ respectively. This value of $h$ and the relative permittivity value of the dielectric material realized are then included into a carefully written visual basic program in the macros of CST MWS Suites 2017 for pattern generation and simulations of the antenna's SMA feed and radiation characteristics looking at the $S_{11}$ values and the directivity of the RLSA antenna with a $100 \mathrm{~mm}$ aperture.

\section{$2.2 S_{11}$ Simulations for a $100 \mathrm{~mm}$ RLSA Aperture}

$\mathrm{S}_{11}$ simulations for a $100 \mathrm{~mm}$ aperture is shown in Figure 4.

CST Simulated S11 Graph

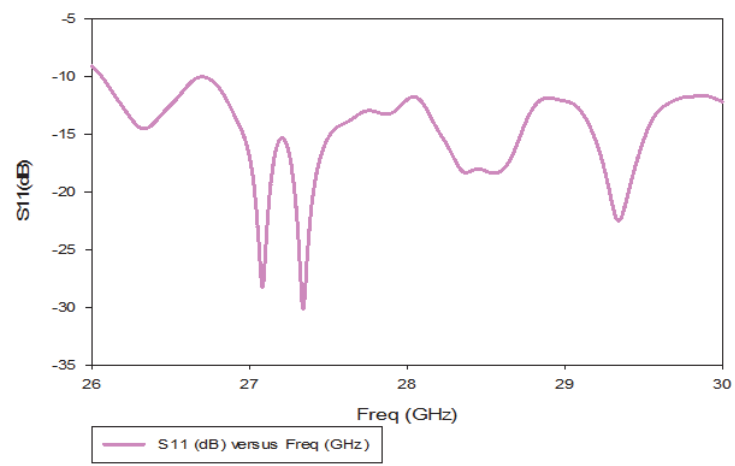

Figure 4. $\mathrm{S}_{11}$ Simulations for a $100 \mathrm{~mm}$ Aperture RLSA 


\subsection{Structure of RLSA Antenna}

The schematics of a standard structure of a single layer RLSA antenna/feeder is shown in figure 5. Excitation of the feeder is a coaxial cable mounted at the rear of the coaxial to waveguide transition feed. Power generated is converted from Transverse Electromagnetic (TEM) transmission line mode to TEM cavity mode, this is made possible with the aid of a disc end probe which matches the radial waveguide to the coaxial transmission line. The radiation from the feed travel radially outward into the cavity.

The Presence of a dielectric material in the cavity creates slow wave thereby minimizing reflection in the coaxial transmission line direction. An area of the radius $\rho$ min at the center on the radiating surface is left without slots so as to create stability of waves arriving from the feeder. The radiating surface is populated with a number of slots that are arranged in such a manner to provide adequate percentage of the energy emanating from the feeding network.

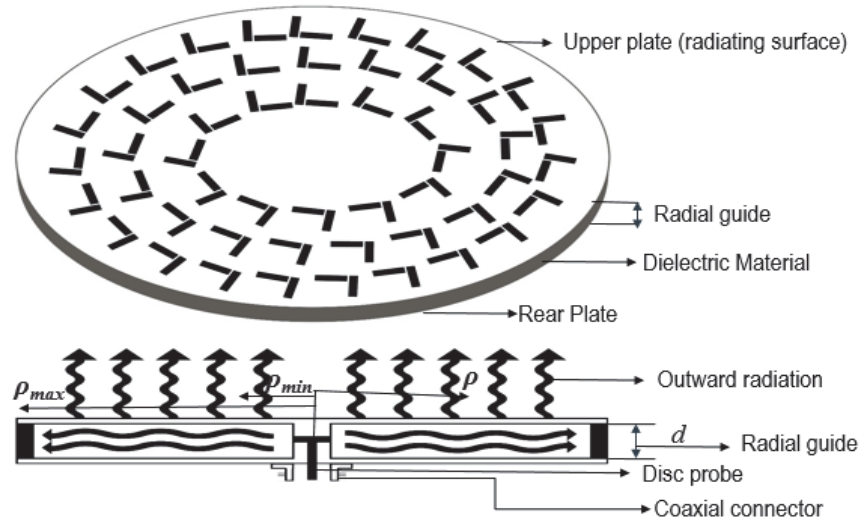

Figure 5. RLSA Antenna Feeder and Radiating Surface. Beheshti-Zavareh \& Hakkak (2009)

Polypropylene was used for the study as dielectric material with relative permittivity value of 1.0 which was obtained from the brainstorm optimization algorithm, this dielectric value was deposited in the visual basic program in the macros of CST MWS Suite 2017.The design is then simulated in other to investigate possible improvement in bandwidth and radiation efficiency parameters of the antenna.

\section{Results}

The radiation performance on simulation in CST is as presented in Figure 6 while the Table 3 gives a comparative summary of results obtained from previous work and the results obtained using the BSO algorithm in terms of bandwidth, directivity, gain and return loss values.

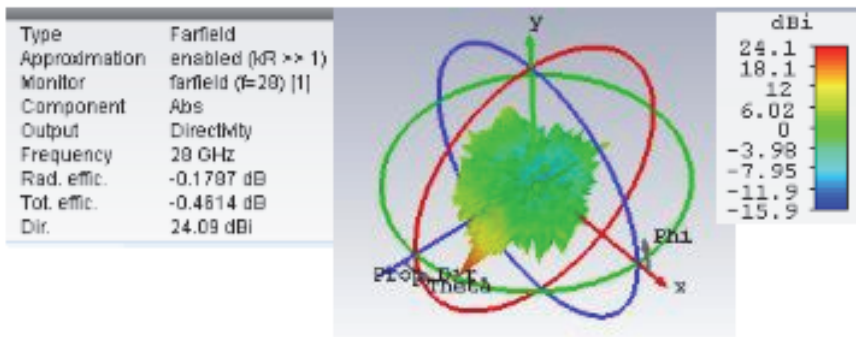

Figure 6. Radiation performance CST perspective

Table 3. Comparative Analysis of the Results

\begin{tabular}{lcc}
\hline Radiation Parameters & Maina, I., et al (2016) & BSO Study [2018] \\
\hline Frequency $(\mathrm{GHz})$ & 28.00 & 28.00 \\
Directivity $(\mathrm{dBi})$ & 18.40 & 24.09 \\
$\mathrm{~S}_{11}(\mathrm{dBi})$ & -12.12 & -12.32
\end{tabular}




$\begin{array}{lll}\text { Impedance Bandwidth }(\%) & 58.50 & 99.61 \\ \text { Rad. Efficiency }(\%) & 96.00 & 95.97 \\ \text { Dish Size }(\mathrm{mm}) & 100.0 & 100.0 \\ \text { Squint Angle }(\mathrm{Deg}) & 20.00 & 20.00 \\ \text { Dielectric }(\varepsilon r) & 2.220 ; 2.230 & 1.800 \\ \text { Gain }(\mathrm{dBi}) & 18.13 & 23.09\end{array}$

In the Table 3, comparative performance in Ka Band between previous research conducted in downlink Ka Band at $28 \mathrm{GHz}$ is shown. The antenna diameter of some research conducted and the current work at $100 \mathrm{~mm}$ respectively. The reflection coefficient performance from previous work and the respective equivalent directivities compared with the current work are equally shown. The table clearly highlight improvements achieved with the use of BSO technique for the same antenna diameter which provides about $4 \mathrm{dBi}$ improvement in the value of directivity on the same Ka Band at $28 \mathrm{GHz}$.

\section{Conclusions}

The BSO algorithm as seen from this submission has been able to eradicate design time wastage associated with feeder optimal parameter selections for RLSA antennas at $28 \mathrm{GHz}$ thus guaranteeing better radiation characteristics.

The work thus affirms the BSO as a feasible tool for feeder design optimization because of the optimal feeder values obtained that resulted in better radiation characteristics from CST MWS simulations at $28 \mathrm{GHz}$.

\section{References}

Ando, M., Sakurai, K., \& Goto, N. (1986). Characteristics of a radial line slot antenna for $12 \mathrm{GHz}$ band satellite TV reception. IEEE Transactions on Antennas and Propagation, 34(10), 1269-1272.

Beheshti-Zavareh, O., \& Hakkak, M. (2009). A stable design of coaxial adaptor for Radial Line Slot Array Antenna, pp. 51-62. https://doi.org/10.2528/PIER08071902

Davis, P. (2000). A linear polarised radial line slot array antenna for direct broadcast satellite services. $\mathrm{PhD}$ Thesis submitted to the department of Computer Science and Electrical Engineering, University of Queensland, Australia.

Davis, P. W. (1997). Experimental investigations into a linearly polarized radial slot antenna for DBS TV in Australia. IEEE Trans. Antennas Propag., 45(7), 1123-1129. https://doi.org/10.1109/8.596903

Davis, P. W., \& Bialkowsky, M. E. (1999). Linearly Polarized Radial-line Slot-array Antennas with Improved Return-loss Performance. IEEE Antennas and Propagation Magazine," vol. 41, no. 1, 1999.DOI:10.1109/74.55027

Davis, P. W., \& Bialkowsky, M. E. (1997). The performance of a linearly polarised RLSA antenna for different beam squint angles. Microw. Conf. Proc. APMC'97, Asia-Pacific. IEEE. https://doi.org/10.1109/APMC.1997.654626

Davis, P. W., Bialkowski, M. E., \& Lucia, S. (2000). Beam Synthesis in Linearly Polarized Radial Line Slot Array Antennas, 94-97.

Hosseini, A., Kabiri, S. E., Komaros, F. D., \& Capolino, F. (2016). High gain single -layer radial line slot array designed for v-band applications. In 2016 IEEE International Symposium on Antennas and Propagation (APSURSI), pp. 1403-1404.

Maina, I., Rahman, T. A., Khalily, M., \& Zakwoi. S. I. (2016). Multilayered Cavity Material Radial Line Slot Array Antenna with Improved Bandwidth for 5G Communication Application. Mod. Appl. Sci., 10(8), 134. https://doi.org/10.5539/mas.v10n8p134

Purnamirza, M. J., \& Rahman, T. (2002). The extreme beamsquint technique to minimize the reflection coefficient of very small aperture radial line slot array antennas. Electromagn. Waves Appl., 2267-2276. https://doi.org/10.1080/09205071.2012.733491

Shi, Y. H. (2011). An optimization algorithm based on brainstorming process. Int. J. Swarm Intell Res., 2(4), 3562. https://doi.org/10.4018/978-1-4666-2479-5.ch008

Soyinka, O. K., \& Duan, H. B. (2016). Optimal Impulsive Thrust Trajectories for Satellite Formation via Improved Brainstorm Optimization, In: Tan Y., Shi Y., Niu B. (eds) Advances in Swarm Intelligence. ICSI 2016. Lecture Notes in Computer Science, 9712, 491-499. Springer, Cham. https://doi.org/10.1007/978-3-319-41000-5_49 
Soyinka, O. K., \& Duan, H. B. (2017). Satellite formation keeping via chaotic artificial bee colony. Aircr. Eng. Aerosp. Technol., 89(2), 246-256. https://doi.org/10.1108/AEAT-02-2014-0019

Sun, C. H., \& Duan, H. B (2013). Optimal Satellite Reconfiguration Based on Closed-loop Brain Storm Optimization. IEEE Computational Intelligence Magazine, 8(4), 59-51. https://doi.org/10.1109/MCI.2013.2279560

Suryana, J., \& Kusuma, D. B. (2015). Design and Implementation of RLSA antenna for mobile DBS Applications in Ku-band downlink direction. In 2015 international Conference on Electrical Engineering and Informatics (ICEEI), 341-345.

Takahashi, M., Takada, J. I., Ando, M., \& Goto, N. (1999). A slot design for uniform aperture field distribution in single-layered radial line slot antennas. IEEE Trans. Antennas Propag., 39(7), 954-959). https://doi.org/10.1007/978-981-4560-75-7_62-1

Yamamoto, N. I., Ono, T. H., Ando, M., \& Goto, N. (1999). Near field distributions in radial line slot antennas for surface wave coupled plasma generation. Antennas Propag. Soc. Int. Symp. IEEE, 1999. https://doi.org/10.1109/APS.1999.789478

Zakwoi, S. I., Rahman, T. A., Maina, I., \& Elijah, O. (2015). Design of ka band downlink radial line slot array antenna for direct broadcast satellite services. 2014 IEEE Asia-Pacific Conf. Appl. Electromagn. APACE 2014 - Proceeding, (pp. 159-162). https://doi.org/10.1109/APACE.2014.7043767

Zhan, Z., Zhang, J., Shi, Y. H., \& Liu, H. (2012). A Modified Brain Storm Optimization. IEEE Australia World Congress on Computational Intelligence 2012 Proceeding, 1-8. https://doi.org/10.1109/CEC.2012.6256594

\section{Copyrights}

Copyright for this article is retained by the author(s), with first publication rights granted to the journal.

This is an open-access article distributed under the terms and conditions of the Creative Commons Attribution license (http://creativecommons.org/licenses/by/4.0/). 